\title{
Clinical Study \\ Predictors of Serum Total IgE in a Random Sample of 7-17 Year Old Children
}

\author{
Sofie Strømgaard, ${ }^{1}$ Simon Francis Thomsen, ${ }^{2}$ Mogens Fenger, ${ }^{3}$ and Vibeke Backer ${ }^{1}$ \\ ${ }^{1}$ Department of Respiratory Medicine, Bispebjerg Hospital, 2400 Copenhagen, Denmark \\ ${ }^{2}$ Department of Dermatology, Bispebjerg Hospital, 2400 Copenhagen, Denmark \\ ${ }^{3}$ Department of Clinical Biochemistry, Hvidovre Hospital, 2650 Hvidovre, Denmark \\ Correspondence should be addressed to Simon Francis Thomsen, sft@city.dk
}

Received 7 March 2011; Accepted 11 April 2011

Academic Editors: A. Fukushima and A. S. Zacharasiewicz

Copyright ( $\odot 2011$ Sofie Strømgaard et al. This is an open access article distributed under the Creative Commons Attribution License, which permits unrestricted use, distribution, and reproduction in any medium, provided the original work is properly cited.

\begin{abstract}
Background. There is little knowledge of the causes for raised serum total immunoglobulin E (IgE) in children. We investigated the association between perinatal, socioeconomic, demographic, clinical, paraclinical, and early life factors, and total serum IgE. Methods. In a random sample of 7-17 year old children, data on possible risk factors were collected using a questionnaire and total serum IgE was measured in venous blood. Airway responsiveness to inhaled histamine was measured and skin prick tests were performed using common aeroallergens. Data was analysed using linear regression. Results. Positive skin prick test $(P<.001)$, airway hyperresponsiveness $(P=.003)$, atopic dermatitis $(P=.046)$, and parental predisposition to allergy $(P=.047)$ showed a significant relation to serum total IgE levels. There was no association of smoking to serum total IgE. Conclusions. This study showed a strong positive relationship between markers of the atopic syndrome and serum total IgE levels in children. In contrast to some earlier studies, we did not find a significant relation between smoking status and IgE.
\end{abstract}

\section{Introduction}

There is only limited knowledge about factors that cause increased serum total immunoglobulin E (IgE) in children. Generally, IgE has been found to be higher in men than in women $[1,2]$. However, some studies found this difference only among smokers $[3,4]$, whereas others have found that the difference was confined to the age group above 55 years $[5,6]$. Serum total IgE has been shown to decrease with age with the highest concentrations found in children and adolescents $[2,5,7]$. Some investigations have found this association only in different subgroups of the study populations but results are contradictory $[3,4,6,8]$. The association between allergic symptoms and serum total IgE has been found to be dependent on atopic status $[4,9,10]$. Notably, subjects with positive skin prick test have higher IgE levels $[4,7]$. Rhinitis, wheeze, and current asthma are closely associated with $\operatorname{IgE}$ [10]. Furthermore, subjects with occupational exposure to dust or gas have been shown to have higher IgE levels than those not exposed [6]. Most of these studies were on adult populations, whereas some also included children above the age of 15. One study included children older than 11 years and three included children older than 6 years. In one-year olds, IgE has been found to be associated with parental IgE levels [11], whereas some studies have found an effect of alcohol intake on serum total IgE in adults [12]. Smoking has been shown to increase IgE in both men and women [1, $2,6,9]$ but especially in men $[3,8]$. Warren et al. found that smoking was associated with increased IgE only in men [8], whereas another study found that the association between smoking and increased IgE was present only in subjects older than 20 years of age [4]. Due to the relatively sparse knowledge of predictors of serum total IgE in children we studied the association between perinatal, demographic, socioeconomic, clinical, paraclinical and early life factors, and serum total IgE in a population sample of children aged 7-17 years.

\section{Methods}

2.1. Subjects. A random sample of children and adolescents living in Copenhagen, Denmark was invited to take part in a clinical examination [13]. All subjects were drawn at 
random from the civil registration list. The mean age was 12.1 years (age range $7-17$ years, $S D=2.9$ years). A total of 480 subjects (response rate, $33.3 \%$ ) participated in the survey of whom $222(46.3 \%)$ were males. To examine the representativeness of the examined population, telephone interviews were conducted among 116 randomly selected subjects from the group of nonrespondents. The subjects, interviewed by telephone, did not differ significantly from the subjects originally included with respect to sex, age, or prevalence of asthma, but there were significantly fewer children with symptoms of hay fever among the group of nonrespondents.

2.2. Interview and Questionnaire. Information on possible risk factors was collected by means of a parental multiitem questionnaire. The questions were answered in the participating families' homes prior to testing and in cases of doubt consensus was reached with a physician on the day of testing. The exposures assessed included duration exclusively breastfed, type and duration of supplementation with formula feeding, a diagnosis of wheezy bronchitis before two years of age, systemic antibiotics given before age two, a history of maternal smoking during pregnancy, and exposure to domestic passive smoking in the first year of life.

Parental atopic disease was defined as symptoms of hay fever and/or asthma in at least one parent when exposed to allergens encountered in the standard testing panel described below. This method is comparable with the one used in the ECRHS [14]. Symptoms among participants were assessed with the questions: "have you ever had hay fever?", "have you ever had episodes of runny or stuffy nose or sneezing when not having a cold?", "have you ever had asthma?", and "have you ever had episodes of wheezing with shortness of breath?" This information was obtained on the day of examination by interview with a physician. Consensus was reached with the accompanying parent. Clinical disease was defined as being present when a positive answer was provided to at least one of the two questions on hay fever and/or the two questions on asthma.

2.3. Serum Total IgE and Skin Prick Tests. Levels of serum total IgE were measured with an enzyme-linked immunosorbent assay (ELISA, on Immulite 2500, DPC, New York, USA). Results were expressed as KIU/L. Values above $150 \mathrm{KIU} / \mathrm{L}$ were regarded as elevated.

Skin prick tests (SPT) were performed using standard dilutions of ten common aeroallergens. The allergens used were birch, grass, mugwort, horse, dog, cat, house dust mite (Dermatophagoides pteronyssinus and Dermatophagoides farinae), and two moulds (Altanaria iridis and Cladosporium herbarium) with positive and negative references being histamine $10 \mathrm{mg} / \mathrm{mL}$ in $50 \%$ glycerol and glycerol $50 \%$, respectively. The concentrations of allergens were $10 \mathrm{HEP}$ (Soluprick SQ system, ALK Albelló, Denmark). Reactions were read after $15 \mathrm{~min}$. A positive test result was defined as a positive reaction to at least one of the allergens. The reaction to each of the allergens was regarded as positive if the mean wheal diameter $((D 1+D 2) \times 1 / 2)$ was at least $3 \mathrm{~mm}$. The participants were told to stop using medications that contained antihistamine at least three days before skin testing.

2.4. Bronchial Responsiveness Test. The method of Yan et al. was used for measuring airway responsiveness to inhaled histamine [15]. Each aerosol was inhaled starting with saline and followed by increasing doses of histamine until a cumulative dose of $7.8 \mu \mathrm{mol}$ had been reached. The test was terminated when the maximum concentration had been reached or when a $20 \%$ decline in $\mathrm{FEV}_{1}$ had occurred before the end of the dosing regimen. For all subjects experiencing at least a $20 \%$ decline in $\mathrm{FEV}_{1}$ the concentration causing a $20 \%$ fall in $\mathrm{FEV}_{1}\left(\mathrm{PD}_{20}\right)$ was calculated. A positive test result (AHR) was defined as a $\mathrm{PD}_{20}$ below $3.9 \mu \mathrm{mol}$.

2.5. Statistical Analysis. Data were analysed with the statistical package SPSS 16.0 (SPSS, Inc., Chicago, IL, USA). Population characteristics were compared using the unpaired $t$-test for numerical data and the chi-square test for categorical data. To determine the impact of different factors on serum total IgE, we first used univariate linear regression analysis and then multivariate linear regression analysis. The response variable was serum total $\operatorname{IgE}$ and the explanatory variables were different perinatal factors, early life factors, socioeconomic factors, demographic factors, clinical, and paraclinical factors. A stipulation of the linear regression analysis is that for each value of the explanatory variable, the values of the response variable should approximately follow the normal distribution. The $\operatorname{IgE}$ values in our population did not follow the normal distribution, so we used $\log (\mathrm{IgE})$ as the response variable as these data were approximately following the normal distribution. Under the assumption of constant variance, all factors examined were analysed by univariate regression. Based on the univariate analysis, factors that had a relation to serum total IgE that was statistically significant or approaching significance were included in the multivariate analysis. Factors of which we lacked knowledge in a great number of the participating subjects were excluded from the multivariate regression analysis. As a consequence, the multivariate analysis included the factors: birth weight, birth length, smoking in pregnancy, parental disposition to allergy, sex, age, smoking, FEV/FVC, airway hyperresponsiveness, asthma, hay fever, positive skin prick test, and atopic dermatitis. Subjects, on which we did not have information about all of these factors, were excluded from the multivariate analysis. A total of 378 subjects were included in the final model.

\section{Results}

Serum total IgE measurements were available for 421 children. The median value of serum total $\operatorname{IgE}$ in the population was 41.8 (Figure 1). In girls, the median value was 33.8, range (2.3-5440), whereas in the boys the median value was 57.4, range (2.1-2173). The geometric mean serum total IgE in the population was 49.3 ; 60.4 in boys and 41.0 in girls, $P<.05$. The proportion of subjects having elevated serum total IgE, asthma, hay fever, atopic dermatitis, and positive skin prick 


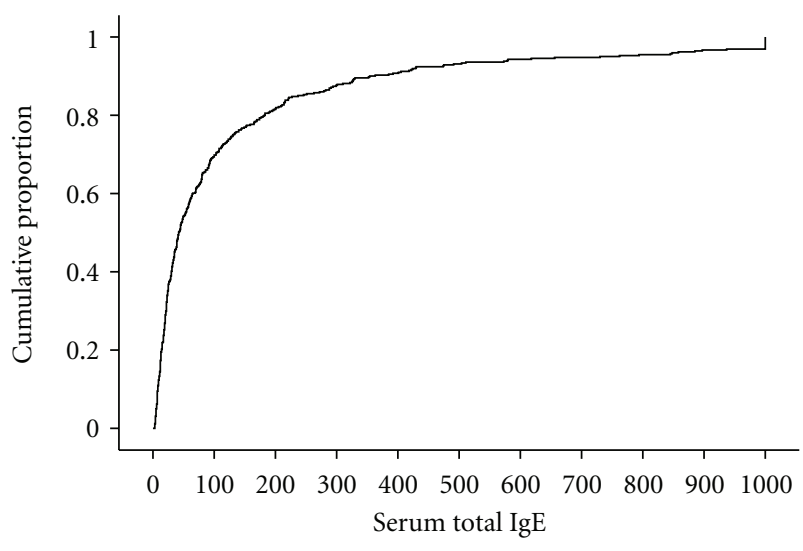

Figure 1

TABLE 1: Population characteristics.

\begin{tabular}{lcccc}
\hline & Boys & Girls & Total & $P$-value \\
& $N=222$ & $N=258$ & $N=480$ & \\
\hline Age & 11.8 & 12.5 & 12.2 & .004 \\
Serum total IgE & 60.4 & 41.0 & 49.3 & .008 \\
High IgE & $53(26.4)$ & $44(20.0)$ & $97(23.0)$ & .121 \\
Asthma & $20(9.0)$ & $24(9.3)$ & $44(9.2)$ & .912 \\
Hay fever & $54(24.3)$ & $58(22.5)$ & $112(23.3)$ & .634 \\
Atopic dermatitis & $57(25.7)$ & $74(28.7)$ & $131(27.3)$ & .461 \\
Positive skin prick test & $50(22.9)$ & $41(16.3)$ & $91(19.4)$ & .068 \\
\hline
\end{tabular}

test was not significantly different between boys and girls (Table 1).

The results of the univariate analyses are shown in Table 2. The following factors were significantly associated with serum total IgE: parental allergic predisposition, early life wheezy bronchitis, male sex, airway hyperresponsiveness, asthma, hay fever, positive skin prick test, and atopic dermatitis. After multivariate adjustment positive skin prick test, airway hyperresponsiveness, atopic dermatitis, and parental predisposition remained significant predictors of serum total IgE (Table 3).

\section{Discussion}

This study showed that positive skin prick test, airway hyperresponsiveness, atopic dermatitis, and parental predisposition to atopic disease were significant predictors of serum total IgE in children, 7-17 years of age. In contrast to some previous studies $[1-3,6,9]$ we did not find any effect of smoking on serum total IgE. However, this is consistent with the findings in a study by Sherril et al. that found an effect of smoking on IgE only among subjects older than 20 years of age [4]. The association between serum total IgE and gender that previous studies have found in adults was not found in this study of children. Also, a decreasing tendency for IgE with age $[2,5,7]$ was not significant in this study, but a comparison with earlier studies is difficult as these mostly included older age groups and a broader range of ages. The association of atopic disease and positive skin prick
TABLE 2: Univariate relationships between different factors and serum total IgE in a sample of children.

\begin{tabular}{|c|c|c|}
\hline Variable & B coefficient & $P$-value \\
\hline \multicolumn{3}{|l|}{ Perinatal factor } \\
\hline Birth weight (per $100 \mathrm{~g}$ ) & 0.103 & .063 \\
\hline Birth length (per cm) & 0.018 & .125 \\
\hline Gestation (per week) & 0.000 & .967 \\
\hline Preterm & -0.002 & .989 \\
\hline Not breastfed & -0.019 & .908 \\
\hline Supplementation & 0.001 & .991 \\
\hline Smoking in pregnancy & 0.066 & .184 \\
\hline Smoking in home & -0.056 & .382 \\
\hline Parental predisposition & 0.133 & .013 \\
\hline Season of birth & & .926 \\
\hline \multicolumn{3}{|l|}{ Early life factors } \\
\hline Pneumonia & 0.128 & .117 \\
\hline Antibiotics & -0.006 & .923 \\
\hline Wheezy bronchitis & 0.319 & .0004 \\
\hline \multicolumn{3}{|l|}{ Socioeconomic factors } \\
\hline Mother age & -0.004 & .509 \\
\hline Mother education & 0.096 & .184 \\
\hline Mother alone & -0.001 & .984 \\
\hline Mother smoke & -0.107 & .130 \\
\hline Father age & -0.010 & .110 \\
\hline Father education & 0.078 & .361 \\
\hline Father smoke & 0.006 & .941 \\
\hline Household income & 0.012 & .539 \\
\hline \multicolumn{3}{|l|}{ Demographic factors } \\
\hline Sex & 0.168 & .008 \\
\hline Age (per year) & -0.018 & .101 \\
\hline BMI (per unit) & 0.006 & .532 \\
\hline Smoking & -0.217 & .062 \\
\hline \multicolumn{3}{|c|}{ Clinical and paraclinical factors } \\
\hline FEV/FVC & -0.825 & .130 \\
\hline AHR & 0.413 & .000002 \\
\hline Asthma & 0.279 & .011 \\
\hline Hay fever & 0.387 & .000000 \\
\hline Positive skin prick test & 0.761 & .000000 \\
\hline Atopisk dermatitis & 0.264 & .0002 \\
\hline
\end{tabular}

TABLe 3: Significant predictors of serum total IgE in a random sample of children, 7-17 years of age.

\begin{tabular}{lccc}
\hline Variable & B coefficient & Standard error & $P$-value \\
\hline Positive skin prick test & 0.663 & 0.078 & $<.001$ \\
Airway hyperresponsiveness & 0.248 & 0.084 & .003 \\
Atopic dermatitis & 0.133 & 0.066 & .046 \\
Parental predisposition & 0.100 & 0.050 & .047 \\
\hline
\end{tabular}

test to serum total IgE level is consistent with the results of earlier studies $[4,7,9,10]$. On the contrary we did not find a significant association between asthma and serum total IgE but this was probably due to a significant effect of airway 
hyperresponsiveness and a strong correlation between this variable and asthma.

The participation rate in this study was quite low. We tried to address this possible bias by making telephone interviews with a random sample of nonparticipants. This showed a comparable distribution of nonparticipants and participants with respect to sex and age but a slightly lower prevalence of hay fever among nonparticipants. A possible parental recall bias cannot be precluded as parents of atopic children may be more aware of factors that could have influenced the development of the disease [16]. Particularly, parents with confirmed or suspected atopic disease may change behaviour during pregnancy or in early life of the child to try to avoid passing on the disorder [17]. Parental predisposition in this study was defined as symptoms of asthma and/or hay fever in at least one parent when exposed to allergens. This may not be an entirely genetic predisposition as social and environmental factors causing or contributing to the parents' disease could be shared with the child.

In conclusion, these results show that serum total $\operatorname{IgE}$ in children is significantly associated with positive skin prick test, parental predisposition to atopic disease, airway hyperresponsiveness, and atopic dermatitis.

\section{Conflicts of interest}

The authors declared that there are no conflicts of interest.

\section{References}

[1] B. Wüthrich, C. Schindler, T. C. Medici, J. P. Zellweger, and P. Leuenberger, "IgE levels, atopy markers and hay fever in relation to age, sex and smoking status in a normal adult Swiss population," International Archives of Allergy and Immunology, vol. 111, no. 4, pp. 396-402, 1996.

[2] C. S. Court, D. G. Cook, and D. P. Strachan, "The descriptive epidemiology of house dust mite-specific and total immunoglobin E in England using a nationally representative sample," Clinical and Experimental Allergy, vol. 32, no. 7, pp. 1033-1041, 2002.

[3] E. J. Jensen, B. Pedersen, E. Schmidt, and R. Dahl, "Serum IgE in nonatopic smokers, nonsmokers, and recent exsmokers: relation to lung function, airway symptoms, and atopic predisposition," Journal of Allergy and Clinical Immunology, vol. 90, no. 2, pp. 224-229, 1992.

[4] D. L. Sherrill, M. Halonen, and B. Burrows, "Relationships between total serum IgE, atopy, and smoking: a twenty-year follow-up analysis," Journal of Allergy and Clinical Immunology, vol. 94, no. 6, pp. 954-962, 1994.

[5] R. A. Barbee, M. Halonen, W. Kaltenborn, M. Lebowitz, and B. Burrows, "A longitudinal study of serum IgE in a community cohort: correlations with age, sex, smoking, and atopic status," Journal of Allergy and Clinical Immunology, vol. 79, no. 6, pp. 919-927, 1987.

[6] E. Omenaas, P. Bakke, S. Elsayed, R. Hanoa, and A. Gulsvik, "Total and specific serum IgE levels in adults: relationship to sex, age and environmental factors," Clinical and Experimental Allergy, vol. 24, no. 6, pp. 530-539, 1994.

[7] R. A. Barbee, M. Halonen, M. Lebowitz, and B. Burrows, "Distribution of IgE in a community population sample: correlations with age, sex, and allergen skin test reactivity," Journal of Allergy and Clinical Immunology, vol. 68, no. 2, pp. 106-111, 1981.

[8] C. P. Warren, V. Holford-Strevens, C. Wong, and J. Manfreda, "The relationship between smoking and total immunoglobulin E levels," Journal of Allergy and Clinical Immunology, vol. 69, no. 4, pp. 370-375, 1982.

[9] O. Zetterström, K. Osterman, L. Machado, and S. G. Johansson, "Another smoking hazard: raised serum IgE concentration and increased risk of occupational allergy," British Medical Journal, vol. 283, no. 6301, pp. 1215-1217, 1981.

[10] B. Burrows, M. Halonen, M. D. Lebowitz, R. J. Knudson, and R. A. Barbee, "The relationship of serum immunoglobulin E, allergy skin tests, and smoking to respiratory disorders," Journal of Allergy and Clinical Immunology, vol. 70, no. 3, pp. 199-204, 1982.

[11] H. A. Orgel, R. N. Hamburger, M. Bazaral et al., "Development of IgE and allergy in infancy," Journal of Allergy and Clinical Immunology, vol. 56, no. 4, pp. 296-307, 1975.

[12] A. González-Quintela, C. Vidal, and F. Gude, "Alcoholinduced alterations in serum immunoglobulin e (IgE) levels in human subjects," Frontiers in Bioscience, vol. 7, pp. e234-e244, 2002.

[13] S. F. Thomsen, C. S. Ulrik, K. Larsen, and V. Backer, "Change in prevalence of asthma in Danish children and adolescents," Annals of Allergy, Asthma and Immunology, vol. 92, no. 5, pp. 506-511, 2004.

[14] R. de Marco, C. Pattaro, F. Locatelli, and C. Svanes, "Influence of early life exposures on incidence and remission of asthma throughout life," Journal of Allergy and Clinical Immunology, vol. 113, no. 5, pp. 845-852, 2004.

[15] K. Yan, C. Salome, and A. J. Woolcock, "Rapid method for measurement of bronchial responsiveness," Thorax, vol. 38, no. 10, pp. 760-765, 1983.

[16] M. Kulig, R. Bergmann, G. Edenharter, and U. Wahn, "Does allergy in parents depend on allergy in their children? Recall bias in parental questioning of atopic diseases," Journal of Allergy and Clinical Immunology, vol. 105, no. 2, part 1, pp. 274-278, 2000.

[17] I. Kummeling, C. Thijs, F. Stelma, M. Huber, P. A. Brandt, and P. C. Dagnelie, "Do parents with an atopic family history adopt a 'prudent' lifestyle for their infant? (KOALA Study)," Clinical and Experimental Allergy, vol. 36, no. 4, pp. 489-494, 2006. 


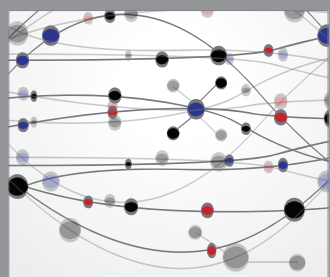

The Scientific World Journal
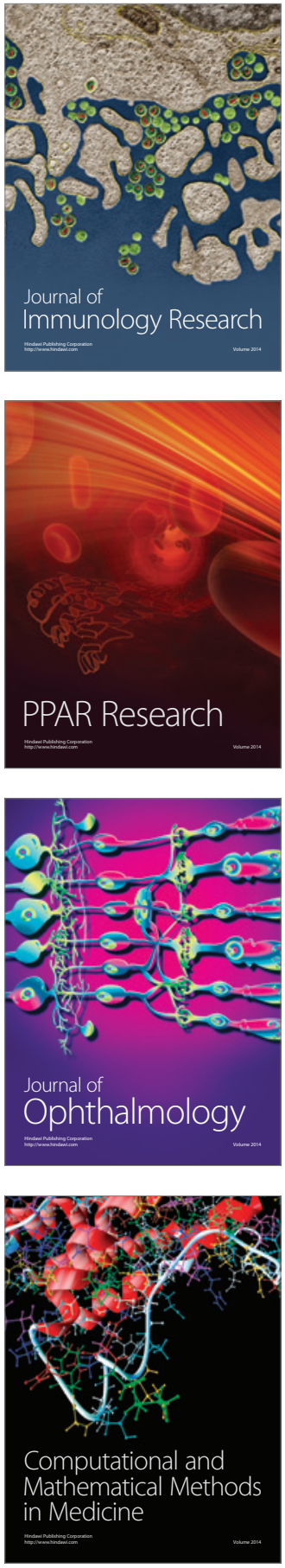

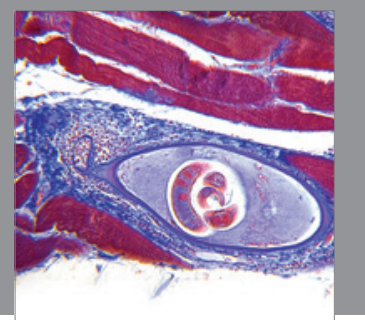

Gastroenterology

Research and Practice
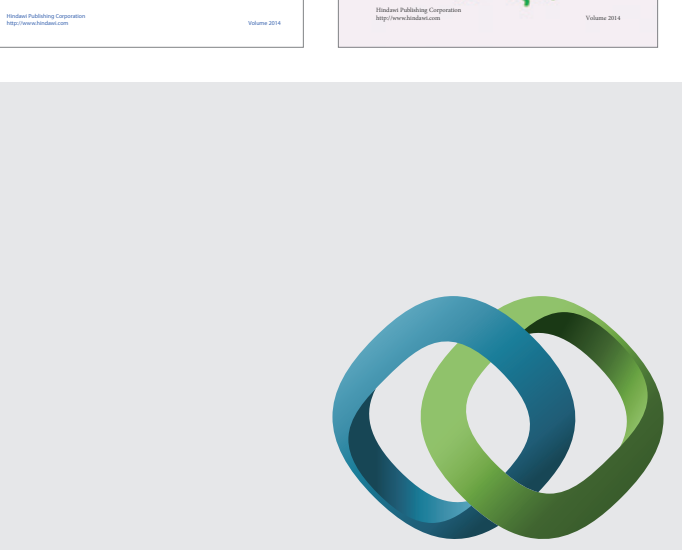

\section{Hindawi}

Submit your manuscripts at

http://www.hindawi.com
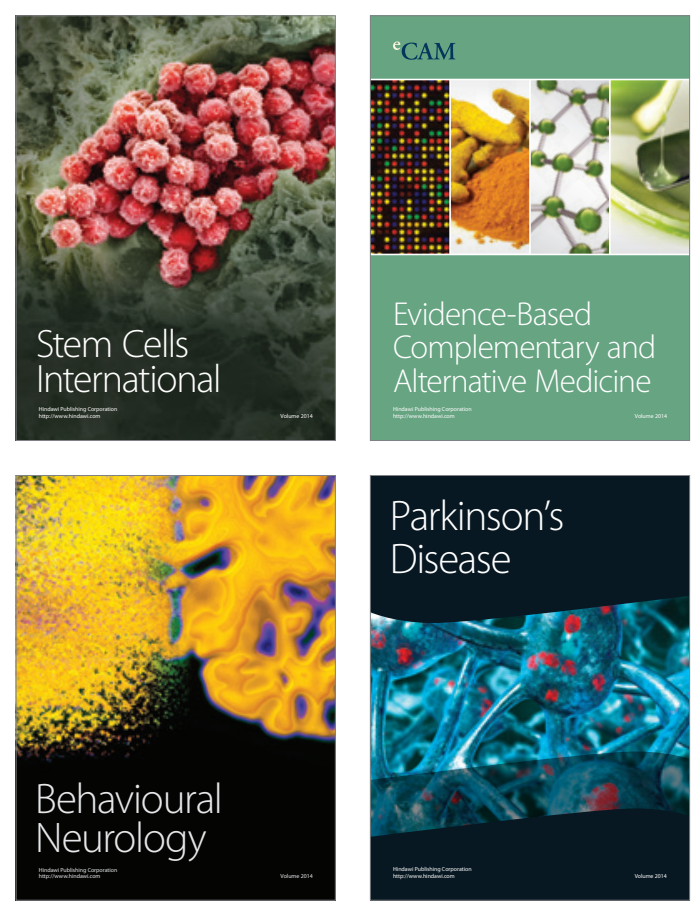

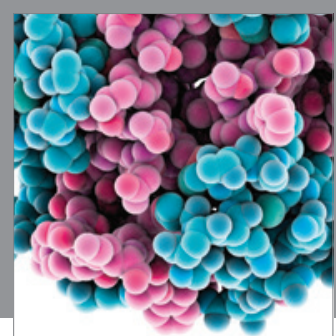

Journal of
Diabetes Research

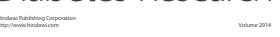

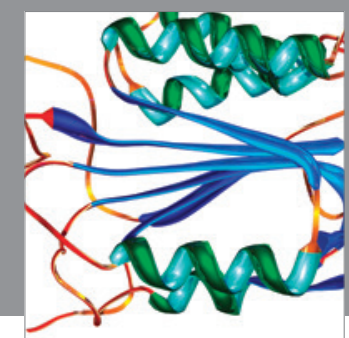

Disease Markers
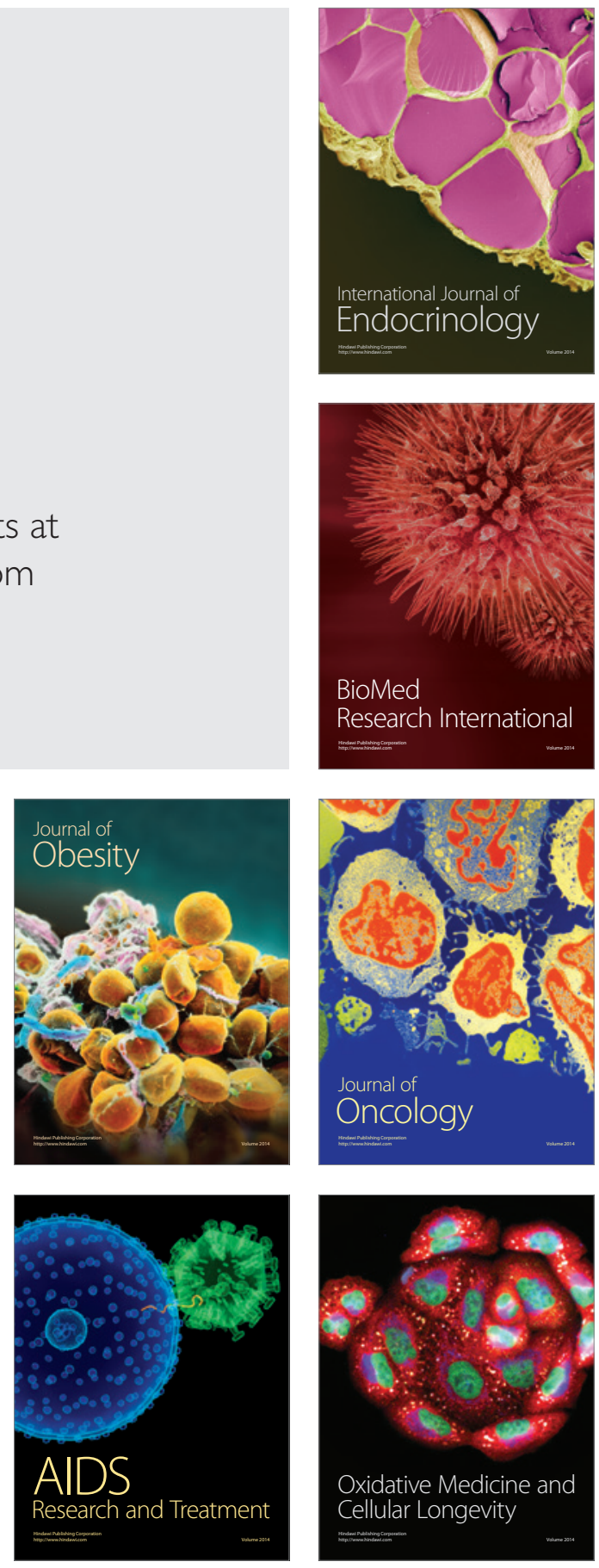